candidate circRNA was performed in vitro and in vivo, including cell viability assay, colony formation assay, cell cycle analysis, apoptosis array and Matrigel migration and invasion assay. Differences between experimental groups and control groups were analysed using the paired t-test and Wilcoxon test.

Results Of the seven-candidate circRNAs, Circ5379-6 was verified to be correlated positively to PPAR $\alpha$. Overexpression of circ5379-6 lead to up-regulation of PPAR $\alpha$ and it thereby suppressed the cell proliferation, inhibited the cell migration and invasion. The most obvious effect of circ5379-6 expression should be the induction of cell apoptosis in the HCC cell lines. Matrix metalloprotein 9 (MMP-9) was down-regulated expressed while Vimentin, N-Cadherin and E-Cadherin were up-regulated expressed in the tumour generated by the cells with circ5379-6 overexpression. Furthermore, the overexpression of circ5379-6 effectively inhibited the tumorigenesis and metastasis of HCC according to the in vivo studies in nude mice.

Conclusions Circ5379-6 acts as an effective tumour inhibitor of HCC via regulating the level of PPAR $\alpha$. It suggests that induction of Circ5379-6 expression may utilise as a potential therapeutic method for HCC.

\section{IDDF2018-ABS-0210 POKEMON OVER-EXPRESSION ACCELERATES THE PROGRESSION OF NAFLD VIA INCREASING LIPID DROPLET DEPOSIT IN HEPATOCYTE}

Jingping Zhou*. Zhongshan Hospital Affiliated to Xiamen University, China

\subsection{6/gutjnl-2018-IDDFabstracts.53}

Background Non-alcoholic fatty liver disease (NAFLD) is considered as the hepatic manifestation of metabolic syndrome and is characterised by the accumulation of lipid droplets. Pokemon (FBI-1/ZATB7A) is an important proto-oncogene which is involved in cancer development and adipogenic gene expression. The aim of our study is to explore the role of Pokemon in the development and progression of NAFLD.

Methods C57BL/6 mice were fed with normal chow (NC) or high-fat diet (HFD) for 16 weeks to induce NAFLD. Pokemon mRNA and protein were detected by RT-PCR and Westernblot as well as immunohistochemistry. NAFLD cell models were established by oleic acid, and si-Pokemon hepatic cancer cell lines were also constructed by plko lentiviral system.

Results Mice fed with HFD for 16 weeks showed increased body weight, liver weight, liver-to-body weight ratio as well as increased lipid accumulation as shown by $\mathrm{H}$ and $\mathrm{E}$ staining and Oil Red $\mathrm{O}$ staining consistent with the establishment of NAFLD. The Pokemon mRNA as determined by RT-PCR and protein expression as determined by Western blot and immunohistochemistry were significantly increased in mice fed with HFD compared with the NC group $(\mathrm{p}<0.01)$. The upregulated Pokemon was accompanied by increased serum TNF- $\alpha$, IL-6, triglyceride, cholesterol and MDA levels in HFD group $(\mathrm{p}<0.01)$. For in vitro study, Pokemon and SREBP-1 protein expression in HepG2 were increased in a concentrationdependent manner when treated with oleic acid $(\mathrm{p}<0.01)$. SREBP-1 and FAS mRNA expression were also increased which could be counteracted by pokemon silencing. Knockdown pokemon by si RNA in HepG2 cells showed decreased lipid accumulation, triglyceride content, suppressed mRNA expression of lipogenic genes including FASN, SREBP, SCD-1,
HMGCR and genes related with oxidation metabolism including Cpt1 and Acadm.

Conclusions Pokemon promotes NAFLD progression via increasing lipid accumulation and repressing free acids oxidation.

\section{IDDF2018-ABS-0216 ANTIDIABETIC EFFECTS OF SODIUM ORTHOVANADATE AND TRIGONELLA FOENUM GRAECUM SEED POWDER IN LIVER OF RAT MODEL}

Pardeep Kumar*, Najma Baquer. School of Life Sciences, Jawaharlal Nehru University, New Delhi, India

\subsection{6/gutjn|-2018-IDDFabstracts.54}

Background Diabetes has been considered as one of the fastest growing epidemic worldwide; the number of people with diabetes is estimated to increase from 381.8 million in 2013 to 591.9 million in 2030. Oxidative stress in diabetic tissues is accompanied by high level of free radicals and the simultaneously declined antioxidant enzymes status leading to cell membrane damage. In the present study, the effect of sodium orthovanadate (SOV) and Trigonella foenum graecum seed powder (TSP) administration has been studied on blood glucose and insulin levels, antioxidant enzymes, lipid peroxidation, lipofuscin and DNA degradation, distribution of glucose transporter (glut 2, glut4) in liver tissues of the alloxaninduced diabetic rats and to see whether the treatment with SOV and TSP is capable of reversing these effects.

Materials and methods Diabetes was induced by administration of alloxan monohydrate $(15 \mathrm{mg} / 100 \mathrm{gm} \mathrm{b.wt}$.) and rats were treated with $2 \mathrm{IU}$ insulin, $0.6 \mathrm{mg} / \mathrm{ml} \mathrm{SOV}, 5 \% \mathrm{TSP}$ in the diet and a combination of $0.2 \mathrm{mg} / \mathrm{ml} \mathrm{SOV}$ with $5 \%$ TSP separately for 21 days. Control animals were given only the vehicle.

Results Diabetic rats showed hyperglycemia with almost fourfold high blood glucose levels. Hyperglycemia increases lipid peroxidation and DNA degradation, causing decreased activities of membrane-bound ATPases, antioxidant enzymes and glucose transporter expression with diabetes in the rat liver. Rats treated with a combined dose of SOV and TSP had glucose levels comparable to controls, similar results were obtained with the activities of antioxidant enzymes, membrane-bound ATPases, DNA degradation, lipid peroxidation and glucose transporter in the liver of diabetic rats.

Conclusions Our results showed that lower doses of vanadate $(0.2 \mathrm{mg} / \mathrm{ml})$ could be used in combination with Trigonella to effectively counter diabetic alterations without any toxic side effects. Therefore combined therapy can indeed be considered a better alternative to being explored further as a means of diabetic control.

\section{IDDF2018-ABS-0232 WEARABLE TECHNOLOGY (MI BAND AND YU BAND) A BOON FOR PATIENTS WITH CHRONIC KIDNEY DISEASE}

Vikas Sharma*, SB Sharma. University College of Medical Sciences (UCMS), India

\subsection{6/gutjnl-2018-IDDFabstracts. 55}

Background New wearable sensor networks together with smartphone applications are being examined and tested for their potential to monitor and manage patients with chronic kidney disease (CKD). To develop methods for analyses and 
monitor of map the intersection(s) of nephrology data in relation to CKD via wearable technology (MI band and Yu band). To study effects of daily life routine activities on data by a wearable device that can obtain real-time CKD data, help technologists understand medical aspects, and clinicians to understand technological processes them and provide assistance based on pre-determined specifications in CKD patients in New Delhi, India.

Methods Total of $78 \mathrm{CKD}$ patients were taken as a subject with an equal ratio of male and female at university college of medical sciences, New Delhi, India. Wearable monitoring devices were put on the wrist of CKD patients for 30 days, and a questionnaire was filled out by each patient. Both diabetes and cardiovascular disease, in turn, are known as important factors for developing CKD and aggravation toward once end-stage renal disease. In all subjects, blood glucose was measured on daily basis with day to day data of their monitoring of step count, blood pressure, calorie burnt, insulin dose, motion time i.e. every time when your body was in motion, sleep monitoring (deep sleep, light sleep, wake up time), monitoring heart rate, cardiac arrhythmias to know daily routines and recording them for health purpose.

Results Present results showed that both wearable device readings showed there was a normal heart rate, more calorie burnt with better control of sugar control and average good sleep count in more physically workout, include walking in stroke patients compared to less physically workout CKD patients, identified by professional physiotherapists. Both device readings showed that after changing lifestyle routine among less physically active CKD patients, their post- CKD events normalise.

Conclusions With this study we show that, by using, these wearable devices ensured online assistive feedback for CKD patients is possible with their health awareness, exercising and motivate further studies.

\section{IDDF2018-ABS-0265 BENEFICIAL EFFECTS OF SILYBUM MARIANUM SEED EXTRACT AGAINST HEPATIC FIBROSIS INDUCED BY CARBON TETRACHLORIDE IN RATS}

Sanjay Kumar*, RK Khanna. Department of Basic and Applied Biology, Vivekananda Global University, Jaipur, Rajasthan, India

\subsection{6/gutjnl-2018-IDDFabstracts.56}

Background Silybum marianum or milk thistle is the most well-researched plant in the treatment of liver disease. Silymarin, derived from the milk thistle plant, silybum marianum, has been used for centuries as a natural remedy for diseases of the liver and biliary tract. The current study aimed to investigate the silybum marianum seed extract as hepatoprotective agent verse hepatic damages caused by carbon tetrachloride $\left(\mathrm{CCl}_{4}\right)$.

Methods Male Wistar albino rats were divided into two equal groups $(n=8)$ and treated as follows: Group 1, kept as control group and orally given saline; Group 2, kept as control positive and were administered daily oral doses of silymarin $(50 \mathrm{mg} / \mathrm{kg})$ daily for 21 days and subsequently injected i.p. with $\mathrm{CCl}_{4}(50 \% \mathrm{v} / \mathrm{v}$ in olive oil; $1 \mathrm{ml} / \mathrm{kg})$ on the $22 \mathrm{nd}$ day. $\mathrm{CCl}_{4}$-induced damages were assessed through liver function markers viz.; alkaline phosphatase (ALP), alanine transaminase (ALT), aspartate transaminase (AST), $\gamma$-glutamyltransferase $(\gamma$ -
GT) and lactate dehydrogenase (LDH). Changes in lipid profile were checked by measuring serum total cholesterol (TC), triglycerides (Tg), high-density lipoproteins (HDL) and lowdensity lipoproteins (LDL). Antioxidant status was checked by the activities of antioxidant enzymes (superoxide dismutase, glutathione peroxidase), DNA damages, malondialdehyde (MDA) and nitric oxide (NO) content. The histopathological changes were observed with Masson staining.

Results Administration $\mathrm{CCl}_{4}$ induced an elevation of serum amino- and glutamyl transferases activities and an increased peroxidation, as well as a decrease of superoxide dismutase and glutathione peroxidase activities in the liver. Administration of $\mathrm{CCl}_{4}$ in rats caused a significant increase in liver function and lipid profile indicating hepatic damages which were restored by co-administration of silymarin. Cellular and DNA damages in hepatic tissues were caused by $\mathrm{CCl}_{4}$ which shown clear hepatic fibrosis in addition to disturbing antioxidant enzyme level. Co-treatment with silymarin regulated these markers of oxidative dysfunctions. Silymarin enhances hepatic glutathione and may contribute to the antioxidant defence of the liver.

Conclusions It may be concluded that silymarin has the ability to reverse $\mathrm{CCl}_{4}$ - induced hepatic damages. Silymarin has been used to treat alcoholic liver disease, acute and chronic viral hepatitis and toxin-induced liver diseases.

\section{Clinical Gastroenterology}

\section{IDDF2018-ABS-0001 FOREIGN BODY INGESTION: 4 CASES WITH DIFFERENT MANAGEMENT APPROACH}

${ }^{1}$ Garima Gupta*, '2Deepak Kumar, ${ }^{2}$ Urmila Jhamb. ${ }^{1}$ Hindu Rao Hospital and NDMC Medical College, New Delhi, India; ${ }^{2}$ Maulana Azad Medical College, New Delhi, India

\subsection{6/gutjn|-2018-IDDFabstracts.57}

Background We managed four cases of ingested foreign bodies located in oesophagus and stomach differently. There are specific guidelines for the management of FB ingested in paediatrics age group depending upon type, size, symptoms and location in the digestive tract.

Methods Our first two cases were of ingested button battery which is blunt or non-sharp type contain corrosives, small in size $(<2 \mathrm{~cm}$ diameter) and were impacted in the oesophagus, both the patients presented to us late by day 4 and 5 . Both presented with symptoms of a cough, dysphagia, vomiting and respiratory distress later diagnosed tracheoesophageal fistula and needed surgical correction. Our third case was a 5 years old patient who had ingested a coin, which is blunt in type, was exactly $2 \mathrm{~cm}$ in diameter and was located in the stomach. As per guidelines he was followed serially for location by $\mathrm{X}$ ray and symptoms but was later removed endoscopically, as coin had not passed the stomach outlet by the 4th week and had a risk of mucosal injury, although the patient was asymptomatic. Our fourth case had ingested small blunt plastic toy part which was initially impacted in oesophagus before referred to us. FB was tried to be removed endoscopically from oesophagus as soon as diagnosed with X-ray but was pushed down to the stomach during the procedure. In this case, the patient was further observed by us since the FB reached the stomach which was later found passed spontaneously in stool on day 6 after endoscopic failure. 